\title{
On piecewise linear cell decompositions
}

\author{
AlEXANDER KiRillov, JR
}

\begin{abstract}
We introduce a class of cell decompositions of PL manifolds and polyhedra which are more general than triangulations yet not as general as CW complexes; we propose calling them PLCW complexes. The main result is an analog of Alexander's theorem: any two PLCW decompositions of the same polyhedron can be obtained from each other by a sequence of certain "elementary" moves.

This definition is motivated by the needs of Topological Quantum Field Theory, especially extended theories as defined by Lurie.
\end{abstract}

\section{$57 \mathrm{Q} 15$}

\section{Introduction}

One of the main tools for studying piecewise-linear manifolds is the notion of triangulation, or more generally, cell complexes formed by convex cells. However, for many purposes this is too restrictive. For example, for any explicit computation of state-sum invariants of 3-manifolds, triangulations turn out to be a very inefficient tool: the number of simplices is necessarily quite large, a cylinder over a triangulated manifold (or, more generally, a product of two triangulated manifolds) does not have a canonical triangulation, etc. Allowing arbitrary convex cells helps but does not solve all the problems: for example, a cell decomposition shown below (which is quite useful for extended topological field theories and 2-categories, as it illustrates a 2-morphism between two 1-morphisms) can not be realized using only convex cells.

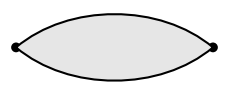

In addition, for many constructions it would be desirable to allow "singular triangulations", where the different faces of the same cell are allowed to be glued to each other (for example, this would allow a cell decomposition of the torus $T^{2}$ obtained by gluing opposite sides of a rectangle). On the other hand, $\mathrm{CW}$ complexes are too general and using them creates other problems: for example, there is no analog of Alexander's theorem describing simple moves necessary to obtain one $\mathrm{CW}$ cell decomposition from another. 
In this note, motivated by the author's earlier work with Balsam [1], we introduce a new notion of a cell decomposition of a compact polyhedron (in particular, a PL manifold) which will address many of the problems mentioned above. We propose calling such cell decompositions PLCW cell decompositions. We also prove an analog of Alexander's theorem: any two PLCW decompositions of the same polyhedron can be obtained from each other by a sequence of certain "elementary" moves; these moves are special cases of cell moves introduced by Oeckl [2].

Acknowledgements The author would like to thank Oleg Viro, Scott Morrison, Benjamin Balsam and Robert Oeckl for helpful suggestions and discussions.

This work was partially suported by NSF grant DMS-0700589.

\section{Basic definitions}

In this section we recall some basic definitions and facts of PL topology, following notation and terminology of Rourke and Sanderson [4], where one can also find the proofs of all results mentioned here.

Throughout this paper, the word "map" will mean "piecewise linear map". We will write $X \simeq Y$ if there exists a PL homeomorphism $X \rightarrow Y$.

For a subset $X \subset \mathbb{R}^{N}$, we denote $\operatorname{Int}(X)$ the interior of $X$, by $\operatorname{cl}(X)$ the closure of $X$ and by $\partial X$ the boundary of $X$. We will also use the following standard notation:

- $\quad B^{n}=[-1,1]^{n} \subset \mathbb{R}^{n}$ is the $n$-dimensional ball.

- $S^{n}=\partial B^{n+1}$ is the $n-$ sphere.

- $\Delta^{n} \subset \mathbb{R}^{n+1}$ is the $n$-dimensional simplex (note that $\Delta^{n} \simeq B^{n}$ ).

For any polyhedra $X \subset \mathbb{R}^{N}$ and a point $a \in \mathbb{R}^{N}$, we denote $a X$ the cone over $X$. More generally, given two polyhedra $X, Y \subset \mathbb{R}^{N}$, we denote by $X Y$ the join of $X, Y$. When using this notation, we will always assume that $X, Y$ are independent, ie that every $p \in X Y$ can be uniquely written as $p=a x+b y, a, b \in \mathbb{R}, a+b=1$. For two polyhedra $X \in \mathbb{R}^{n}, Y \in \mathbb{R}^{m}$, we denote by $X * Y \subset \mathbb{R}^{n+m+1}$ their external join.

We define a convex $n-$ cell $C \subset \mathbb{R}^{N}$ as a convex compact polyhedron generating an affine subspace of dimension $n$; in such a situation, we will also write $\operatorname{dim} C=n$. In Rourke and Sanderson [4], these are called just cells; we prefer a more specific name to avoid confusion with other types of cells to be introduced later. 
For any such cell we can define the set $F(C)$ of faces of $C$ (of arbitrary codimension); each face $F$ is itself a convex cell. We will write $F<C$ if $F, C$ are convex cells and $F$ is a face of $C$.

Recall that each convex cell $C$ is homeomorphic to a ball: $C=\varphi\left(B^{n}\right)$ for some homeomorphism $\varphi$. As usual, we denote

$$
\begin{aligned}
& \stackrel{\circ}{C}=\operatorname{Int}(C)=\varphi\left(\operatorname{Int}\left(B^{n}\right)\right), \\
& \dot{C}=\partial C=\varphi\left(\partial B^{n}\right)
\end{aligned}
$$

if $\operatorname{dim} C>0$. If $\operatorname{dim} C=0$, ie $C$ is a point, then we let $\stackrel{\circ}{C}=C, \dot{C}=\varnothing$.

Following [4], we define a cell complex $K$ as a finite collection of convex cells in $\mathbb{R}^{N}$ such that the following conditions are satisfied:

(1) If $A \in K$ and $B<A$, then $B \in K$.

(2) If $A, B \in K$, and $F=A \cap B \neq \varnothing$, then $F<A, F<B$.

We define the support $|K|=\bigcup_{C \in K} C$; it is a compact polyhedron in $\mathbb{R}^{N}$. Conversely, given a compact polyhedron $X$, a cell decomposition of $X$ is a complex $K$ such that $|K|=X$; it is known that such a decomposition always exists. We will denote by $\operatorname{dim} K$ the dimension of $K$ and by $K^{n}$ the $n$-skeleton of $K$. Given a complex $K$ and a cell $C$, we will denote $K+C$ the complex obtained by adding to $K$ the cell $C$ assuming that it does form a complex.

In particular, given a convex cell $C$, the set $F(C)$ of faces of $C$ is a cell complex, with $|F(C)|=\dot{C}$; by adding to it $C$ itself, we get a cell decomposition of $C$.

\section{Generalized cells}

Let $C$ be a convex cell in $\mathbb{R}^{N}$.

Definition 3.1 A map $f: C \rightarrow \mathbb{R}^{m}$ is called regular if the restriction $\left.f\right|_{C}$ is injective.

Lemma 3.2 If $C$ is a convex cell and $f: C \rightarrow \mathbb{R}^{m}$ is regular, then $C$ admits a cell decomposition $K$ such that for any cell $K_{i} \in K$, the restriction $\left.f\right|_{K_{i}}$ is injective.

Proof By standard results of PL topology, $C$ admits a cell decomposition such that $\left.f\right|_{K_{i}}$ is linear, and a linear map which is injective on an open set is injective.

We can now define the generalization of the notion of a convex cell. 
Definition 3.3 A generalized $n$-cell is a subset $C \subset \mathbb{R}^{N}$ together with decomposition $C=\stackrel{\odot}{C} \sqcup \dot{C}$ such that $\stackrel{\circ}{C}=\varphi\left(\right.$ Int $\left.B^{n}\right), \dot{C}=\varphi\left(\partial B^{n}\right)$ (and thus $C=\varphi\left(B^{n}\right)$ ) for some regular map $\varphi: B^{n} \rightarrow \mathbb{R}^{N}$.

In such a situation, the map $\varphi$ is called a characteristic map.

Note that the definition implies that $C=\operatorname{cl}\left(\stackrel{\circ}{C}^{\circ}\right.$, so $C$ is completely determined by $\stackrel{\circ}{\text {. }}$ It is also clear from Lemma 3.2 that any generalized cell is a compact polyhedron.

Clearly any convex cell is automatically a generalized cell. Other examples of generalized cells are shown in Figure 1 below.

Note that characteristic map $\varphi$ in the definition of generalized cell is not unique. However, as the following theorem shows, it is unique up to a PL homeomorphism of the ball.

Theorem 3.4 Let $C \subset \mathbb{R}^{N}$ be a generalized cell and $\varphi_{1}, \varphi_{2}: B^{n} \rightarrow C$ be two characteristic maps. Then there exists a unique homeomorphism $\psi: B^{n} \rightarrow B^{n}$ such that $\varphi_{1}=\varphi_{2} \circ \psi$.

Proof Since restriction of $\varphi_{i}$ to $\operatorname{Int}\left(B^{n}\right)$ is injective, the composition $\stackrel{\circ}{\psi}=\varphi_{2}^{-1} \varphi_{1}$ is well defined as a map $\operatorname{Int}\left(B^{n}\right) \rightarrow \operatorname{Int}\left(B^{n}\right)$. To show that it can be extended to the boundary, note that it follows from Lemma 3.2 that one can find a cell decomposition $K$ of $B_{n}$ such that $\left.\stackrel{\circ}{\psi}\right|_{K_{i}}$ is linear for every $n$-cell $K_{i} \in K$. This immediately implies that $\stackrel{\circ}{\psi}$ can be extended to a homeomorphism $\psi: B^{n} \rightarrow B^{n}$.

It is easy to show that cone and join of generalized cells is again a generalized cell. Namely, if $C=\varphi\left(B^{n}\right)$ is a generalized cell, and $a C$ is the cone of $C$, then the map $\varphi$ can be in an obvious way lifted to a map $\{\mathrm{pt}\} * B^{n} \simeq B^{n+1} \rightarrow a C$, which is easily seen to be regular. Thus, $a C$ is a generalized cell. In the similar way, using homeomorphism $B^{m} * B^{n} \simeq B^{m+n+1}$, one shows that if $C_{1}, C_{2}$ are generalized cells that are independent, then the join $C_{1} C_{2}$ is also a generalized cell.

\section{Generalized cell complexes}

From now on, unless noted otherwise, the word "cell” stands for a generalized cell.

Definition 4.1 A generalized cell complex (g.c.c.) is a finite collection $K$ of generalized cells in $\mathbb{R}^{N}$ such that

(1) for any distinct $A, B$ in $K$, we have

$$
\stackrel{\circ}{A} \cap \stackrel{\circ}{B}=\varnothing .
$$

(2) for any cell $C \in K, \dot{C}$ is a union of cells. 
Support $|K| \subset \mathbb{R}^{N}$ of a generalized cell complex $K$ is defined by

$$
|K|=\bigcup_{C \in K} C
$$

A generalized cell decomposition of a compact polyhedron $P \subset \mathbb{R}^{N}$ is a generalized complex $K$ such that $|K|=P$.

We define the dimension $\operatorname{dim} K$ of a generalized cell complex and the $n$-skeleton $K^{n}$ in the usual way. Also, if $A, B \in K$ are cells such that $A \subset \dot{B}$, we will say that $A$ is a face of $B$ and write $A<B$; clearly this is only possible if $\operatorname{dim} A<\operatorname{dim} B$.

If $K, L$ are g.c.c., we denote by $K+L$ the complex obtained by taking all cells of $K$ and all cells of $L$, assuming that the result is again a g.c.c.

Example 4.2 (1) Any cell complex is automatically a g.c.c.

(2) A 0-dimensional g.c.c. is the same as finite collection of points. A 1-dimensional g.c.c. is the same as a finite collection of points (vertices) and nonintersecting arcs (1-cells) with endpoints at these vertices. Note that loops are allowed.

(3) Figure 1 shows some examples of 2-dimensional g.c.c.

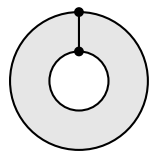

(a)

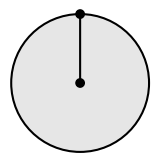

(b)

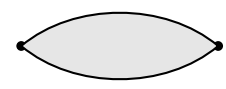

(c)

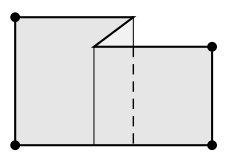

(d)

Figure 1. Examples of 2-dimensional generalized cell complexes. The last one can be visualized as a sheet of paper with a fold, with the lower edge glued back to itself. Note that it only has four 1-cells: the lines showing where the paper was folded are not 1-cells.

(4) Figure 2 shows a generalized cell decomposition of $S^{1} \times I \times I$ consisting of a single 3 -cell, five 2 -cells, eight 1 -cells and 4 vertices.

Definition 4.3 Let $K, L$ be g.c.c. A regular cellular map $f: L \rightarrow K$ is a map $f:|L| \rightarrow|K|$ such that for every cell $C \in L, C=\varphi\left(B^{n}\right)$, there exists a cell $C^{\prime} \in K$ such that $C^{\prime}=f(C)$ and moreover, $f \circ \varphi: B^{n} \rightarrow C^{\prime}$ is a characteristic map for $C^{\prime}$.

In other words, such a map is allowed to identify different cells of $L$ but is injective on the interior of each cell.

An example of a regular cellular map is shown in Figure 3. 


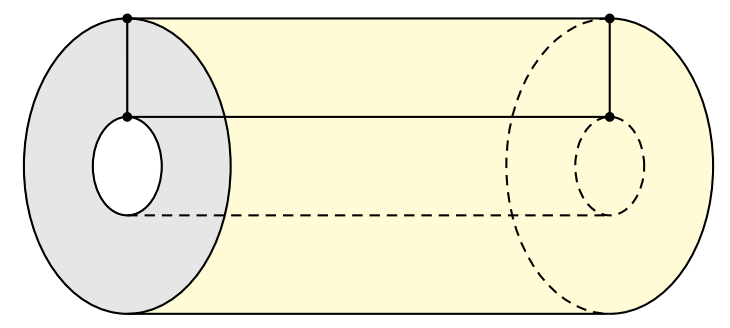

Figure 2. A generalized cell decomposition of $S^{1} \times I \times I$

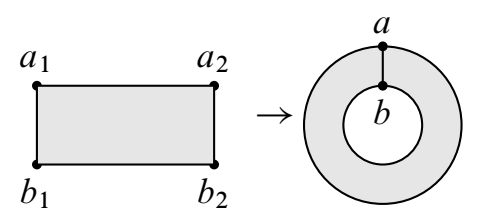

Figure 3. An example of a regular cellular map. It identifies edges $a_{1} b_{1}$ and $a_{2} b_{2}$, sending each of them to edge $a b$.

\section{PLCW complexes}

In this section, we give the central definition of the paper.

Definition 5.1 A generalized cell complex (respectively, a generalized cell decomposition) $K$ will be called a PLCW complex (respectively, PLCW decomposition) if $\operatorname{dim} K=0$, or $\operatorname{dim} K=n>0$ and the following conditions hold:

(1) $K^{n-1}$ is a PLCW complex.

(2) For any $n$-cell $A \in K, A=\varphi\left(B^{n}\right)$, there exists a PLCW decomposition $L$ of $\partial B^{n}$ such that the restriction $\left.\varphi\right|_{\partial B^{n}: L \rightarrow K^{n-1}}$ is a regular cellular map. (It follows from Theorem 3.4 that this condition is independent of the choice of characteristic map $\varphi$.)

In other words, a PLCW is obtained by successively attaching balls, and the attaching map should be a regular cellular map for some PLCW decomposition of the boundary sphere.

Note that this definition is inductive: definition of an $n$-dimensional PLCW complex uses definition of an (n-1)-dimensional PLCW complex.

Example 5.2 Among examples in Example 4.2, example 2(d) is not a PLCW complex. All other are PLCW. 
It is easy to show that for an $n$-cell $A \in K$ and fixed choice of characteristic map $\varphi: B^{n} \rightarrow A$, the generalized cell decomposition $L$ of $\partial B^{n}$ used in Definition 5.1 is unique. Indeed, the cells of $L$ are closures of connected components of $\varphi^{-1}\left(\stackrel{\circ}{K}_{i}\right)$, $K_{i} \in K^{n-1}$. We will call such an $L$ the pullback of $K$ under the map $\varphi$ and denote it by

$$
L=\varphi^{-1}(K) .
$$

The following properties of PLCW complexes are immediate from the definition.

(1) $|K|=\bigsqcup_{C \in K} \stackrel{\circ}{C}$.

(2) If $A, B \in K$ are two cells, then $A \cap B$ is a union of cells of $K$.

(3) For any $n$-cell $C \in K, \dot{C}$ is a union of (n-1)-cells of $K$.

(4) Every PLCW complex is automatically a CW complex.

Note that not every CW complex is a PLCW complex, even if its cells are polyhedra. For example, property (3) could fail for more general CW complexes.

The following two lemmas, proof of which is straightforward and left to the reader, show that product and join of PLCW complexes is a PLCW complex.

Lemma 5.3 Let $K, L$ be PLCW complexes in $\mathbb{R}^{M}, \mathbb{R}^{N}$ respectively. Define the complex

$$
K \times L=\sum K_{i} \times L_{j} \subset \mathbb{R}^{M} \times \mathbb{R}^{N} .
$$

Then $K \times L$ is a PLCW complex with support $|K| \times|L|$.

Lemma 5.4 Let $K, L$ be PLCW complexes in $\mathbb{R}^{N}$ such that $|K|,|L|$ are independent: every point $p \in|K||L|$ can be uniquely written in the form $p=a x+b y, x \in|K|$, $y \in|L|, a, b \geq 0, a+b=1$. Define the join of them by

$$
K L=K+L+\sum K_{i} L_{j}, \quad K_{i} \in K, L_{j} \in L .
$$

Then $K L$ is a PLCW complex with support $|K||L|$.

The proof is straightforward and left to the reader.

Note that in the case $K=\{a\}$ is a point, we see that the cone

$$
a L=a+L+\sum a L_{i}, \quad L_{i} \in L
$$

of a PLCW complex is a PLCW complex. 


\section{Subdivisions}

Definition 6.1 Let $K, L$ be PLCW complexes. We say that $L$ is a subdivision of $K$ (notation: $L \triangleleft K)$ if $|K|=|L|$ and for any cell $C \in K$, we have $\stackrel{\circ}{C}=\bigcup \stackrel{\circ}{L}_{i}$ for some collection of cells $L_{i} \in L$.

Note that this implies that any cell $L_{i} \in L$ is a subset of one of the cells of $K$ (which is the usual definition of subdivision of cell complexes). Moreover, it is easy to see that if $K, L$ are cell complexes, then this definition is actually equivalent to the usual definition of subdivision.

There is a special kind of subdivisions we will be interested in.

Definition 6.2 Let $K$ be a PLCW complex, $C=\varphi\left(B^{n}\right)$ an $n$-cell, $n>0$ and $L=\varphi^{-1}(K)$ the pullback cell decomposition on $\partial B^{n}$ (see (5-1)). We define the radial subdivision of $K$ to be the subdivision obtained by replacing the cell $C$ by the cone PLCW complex $\varphi(O)+\varphi\left(O L_{1}\right)+\cdots+\varphi\left(O L_{k}\right)$, where $L=\left\{L_{1}, \ldots, L_{k}\right\}$ and $O \in \operatorname{Int}\left(B^{n}\right)$ is the origin. (Recall that a cone of a PLCW complex is PLCW complex; see Lemma 5.4.)

Figure 4 shows examples of radial subdivisions.

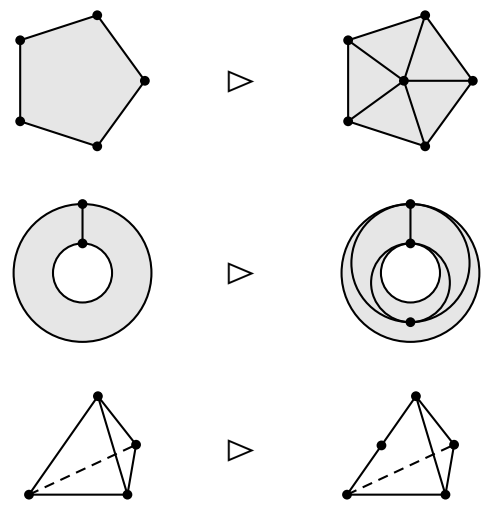

Figure 4. Examples of radial subdivisions. Note that in the last example, we are subdividing a 1 -cell.

Note that this is very closely related to the usual notion of stellar subdivision for simplicial complexes but it is not identical to it. Namely, for radial subdivision we are subdividing just one cell $C$ without changing the higher dimensional cells adjacent to $C$ (see the last example in Figure 4). Comparing it with the definition of the stellar subdivision, we see that if $K$ is a simplicial complex, $C \in K$ an $n$-cell and $L$ is the stellar subdivision of $K$ obtained by starring at $a \in \stackrel{\circ}{C}$, then $L$ can also be obtained by 
(1) replacing $C$ by the radial subdivision $R$.

(2) replacing every cell $A=C B$ in the star of $C$ by the complex $R_{i} B, R_{i} \in R$.

Theorem 6.3 Any PLCW complex $K$ has a subdivision $T \triangleleft K$ which is a triangulation; moreover, $T$ can be obtained from $K$ by a sequence of radial subdivisions.

Proof Let $K^{\prime}$ be obtained from $K$ by radially subdividing of each cell of $K$ of positive dimension in order of increasing dimension. Then it is easy to see that $K^{\prime}$ has the following property:

(6-1) For any $C \in K^{\prime}$, the characteristic map $\varphi: B^{n} \rightarrow C$ is injective.

Now, let $T$ be obtained by again doing the radial subdivision of each cell of $K^{\prime}$ in order of increasing dimension. It is easy to see that $T$ is a triangulation: this follows by induction from the fact that given a triangulation $\dot{T}$ of $S^{n-1}$, the radial subdivision $a \dot{T}$ of $B^{n}$ is a triangulation (which in turn follows from the fact that the cone over a simplex is a simplex).

\section{Elementary subdivisions}

The other type of subdivision will be called elementary subdivision. Informally, these are obtained by dividing an $n$-cell into two $n$-cells separated by an $(n-1)-$ cell. To give a more formal definition, we need some notation.

Let $H_{0} \subset \mathbb{R}^{n}$ be hyperplane defined by equation $x_{n}=0$. It divides $\mathbb{R}^{n}$ into two subspaces:

$$
\begin{aligned}
& H_{+}=\left\{\left(x_{1}, \ldots, x_{n}\right) \in \mathbb{R}^{n} \mid x_{n} \geq 0\right\}, \\
& H_{-}=\left\{\left(x_{1}, \ldots, x_{n}\right) \in \mathbb{R}^{n} \mid x_{n} \leq 0\right\} .
\end{aligned}
$$

For the $n$-ball $B^{n} \subset \mathbb{R}^{n}$, define upper and lower halfballs

$$
B_{+}^{n}=B^{n} \cap H_{+}, \quad B_{-}^{n}=B^{n} \cap H_{-} .
$$

We also define the middle disk and the equator by

$$
B_{0}^{n}=B^{n} \cap H_{0} \simeq B^{n-1}, \quad E=S^{n-1} \cap H_{0} \simeq S^{n-2} .
$$

Lemma 7.1 Let $K$ be a PLCW and $C=\varphi\left(B^{n}\right)$ an $n$-cell. Assume that the pullback decomposition $L=\varphi^{-1}(K)$ of $\partial B^{n}$ is such that the equator $E \subset \partial B^{n}$ is a union of cells of $L$. Let $K^{\prime}$ be the g.c.c. obtained by replacing $C$ by the collection of cells $C_{+}=\varphi\left(B_{+}^{n}\right), C_{-}=\varphi\left(B_{-}^{n}\right), C_{0}=\varphi\left(B_{0}^{n}\right)$.

Then $K^{\prime}$ is a PLCW complex; moreover, $K^{\prime}$ is a subdivision of $K$. 
Definition 7.2 Let $K, K^{\prime}$ be as in Lemma 7.1. Then we say that $K^{\prime}$ is obtained from $K$ by an elementary subdivision of cell $C$; we will also say that $K$ is obtained from $K^{\prime}$ by erasing cell $C_{0}$.

We will write $K \sim_{e} L$ if $K$ can be obtained from $L$ by a finite sequence of elementary subdivisions and their inverses.

Note that elementary subdivisions are essentially the same as $(n, n)$ moves in introduced by Oeckl in [2] and further studied Oeckl's book [3]; in Oeckl's work, these moves are special case of a more general moves called $(n, k)$ moves.

An example of elementary subdivision is shown in Figure 5.

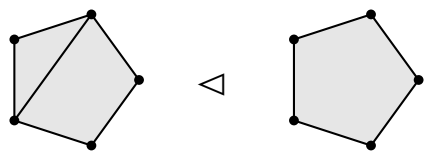

Figure 5. An elementary subdivision

Remark 7.3 Not every subdivision can be obtained by a sequence of elementary subdivisions. For example, the subdivision shown in Figure 6 can not be obtained by a sequence of elementary subdivisions. However, it can be obtained by a sequence of

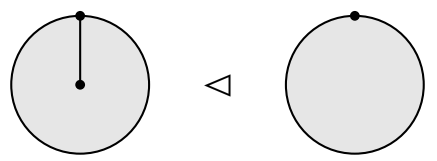

Figure 6. A nonelementary subdivision

elementary subdivisions and their inverses as shown in Figure 7.

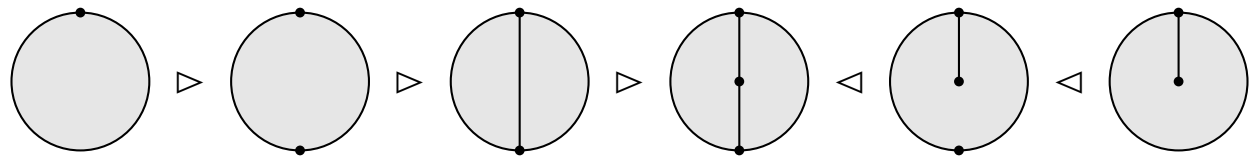

Figure 7. Obtaining a nonelementary subdivision by a sequence of elementary subdivisions and their inverses

Theorem 7.4 If $M=K L$ is a join of two PLCW complexes and $K^{\prime} \triangleleft K$ an elementary subdivision of $K$, then $M^{\prime}=K^{\prime} L$ be obtained from $M$ by a sequence of elementary subdivisions. 
Proof If $C \in K$ is an $n$-cell and $C=C_{+}+C_{-}+C_{0}$ its elementary subdivision as in Lemma 7.1, and $D$ is a cell in $L$, then $C D=C_{+} D+C_{-} D+C_{0} D$ is an elementary subdivision of $C D$, which follows from existence of a homeomorphism $\psi: B^{n} * B^{m} \stackrel{\sim}{\rightarrow}$ $B^{m+n+1}$ such that $\psi\left(B_{0}^{n} * B^{m}\right)=B_{0}^{m+n+1}, \psi\left(B_{ \pm}^{n} * B^{m}\right)=B_{ \pm}^{m+n+1}$. Repeating it for every cell $D \in L$ in order of increasing dimension, we see that $K^{\prime} L$ can be obtained from $K L$ by a sequence of elementary subdivisions.

Corollary 7.5 If $K \sim_{e} K^{\prime}$, then $K L \sim_{e} K^{\prime} L$.

\section{Main theorem}

In this section, we formulate and prove the main theorem of this paper. Recall the notation $K \sim_{e} L$ from Definition 7.2.

Theorem 8.1 Let $K, K^{\prime}$ be two PLCW decompositions of a compact polyhedron $X$. Then $K \sim_{e} K^{\prime}$.

Proof This proves (for PLCW decompositions) the conjecture of Oeck1 [3]: that any cell decompositions can be obtained form each other by a sequence of $(n, k)$ moves; in fact, it proves a stronger result, that $(n, n)$ moves are already enough.

We proceed by induction in $n=\operatorname{dim} X$. If $n=0$, there is nothing to prove. So from now on, we assume that $n>0$ and that the theorem is already proved for all polyhedra of dimension less than $n$.

Step 1 Let $X=B^{n}$ be an $n$-ball, $\dot{K}$ a PLCW decomposition of $S^{n-1}=\partial B^{n}$ and $R=a \dot{K}$ the corresponding radial cell decomposition of $X, a \in \operatorname{Int}\left(B^{n}\right)$. Then $R \sim_{e} B^{n}+\dot{K}$.

Indeed, let $L$ be a PLCW decomposition of $S^{n-1}$ consisting of the upper and lower hemispheres $S_{ \pm}^{n-1} \simeq B^{n-1}$ and some PLCW decomposition $L_{0}$ of the equator $E$. By induction assumption, $\dot{K} \sim_{e} L$; by Corollary 7.5, this implies

$$
a \dot{K} \sim_{e} a L=B_{+}^{n}+B_{-}^{n}+S_{+}^{n-1}+S_{-}^{n-1}+a L_{0} .
$$

By using the induction assumption again, $a L_{0} \sim_{e} B_{0}^{n}+L_{0}$, so $a \dot{K} \sim_{e} B_{+}^{n}+B_{-}^{n}+B_{0}^{n}+S_{+}^{n-1}+S_{-}^{n-1}+L_{0} \sim_{e} B^{n}+S_{+}^{n-1}+S_{-}^{n-1}+L_{0} \sim_{e} B^{n}+\dot{K}$.

Step 2 If $K^{\prime}$ is obtained from $K$ by a sequence of radial subdivisions, then $K^{\prime} \sim_{e} K$. This follows from the previous step and definition. 
Step 3 For any PLCW decomposition $K$, there is a triangulation $T$ such that $K \sim_{e} T$. Indeed, it follows from the previous step and Theorem 6.3.

Step 4 If $T, T^{\prime}$ are triangulations of $X$, then $T \sim_{e} T^{\prime}$.

By Alexander's theorem, $T$ can be obtained from $T^{\prime}$ by a sequence of stellar moves, so it suffices to prove the theorem in the case when $T^{\prime}$ is obtained from $T$ by starring at point $a \in \operatorname{Int}(C)$ for some simplex $C \in T$. By the discussion in Section 6, we can also describe $T^{\prime}$ by replacing $C$ by the radial subdivision $C^{\prime}$ of $C$ and replacing every simplex $A=C B$ in the star of $C$ by $C^{\prime} B$. By Step 2 and Corollary 7.5, this implies that $T^{\prime} \sim_{e} K$.

Combining Steps 3 and 4 above, we arrive at the statement of the theorem.

\section{Orientations}

Recall that the group of homeomorphisms of $B^{n}$ has a homomorphism to $\mathbb{Z}_{2}$, called orientation. Using this, we can define the notion of orientation of a cell.

Definition 9.1 Let $C \subset \mathbb{R}^{N}$ be a generalized $n$-cell. An orientation of $C$ is an equivalence class of characteristic maps $B^{n} \rightarrow C$, where two characteristic maps $\varphi_{1}, \varphi_{2}: B^{n} \rightarrow C$ are equivalent if $\psi=\varphi_{2} \varphi_{1}^{-1}: B^{n} \rightarrow B^{n}$ is orientation-preserving.

An oriented cell $\mathbf{C}=(C,[\varphi])$ is a pair consisting of a cell $C$ and an orientation $[\varphi]$.

Note that any convex $n$-cell $C \subset \mathbb{R}^{n}$ has a canonical orientation. Moreover, if $C \subset \mathbb{R}^{n}$ is a convex $n$-cell, and $D \subset \partial C$ is a generalized $(n-1)-$ cell, then $D$ has a canonical orientation defined by the usual condition:

$$
\varepsilon(\mathbf{C}, \mathbf{D})=1,
$$

where $\varepsilon(\mathbf{C}, \mathbf{D})$ is the incidence number, defined in the same way as for CW cells (see, eg, [4, Appendix A.7]).

Thus, if $C$ is a convex $n$-cell in $\mathbb{R}^{n}$, and $L$ is a PLCW decomposition of $\partial C$, then each of $(n-1)$-cells $L_{i} \in L$ has a canonical orientation.

The following definition generalizes this to an arbitrary oriented cell. 
Definition 9.2 Let $K$ be a PLCW complex, and $\mathbf{C}=(C,[\varphi])$ an oriented cell. Let $L=\varphi^{-1}(K)$ be the pullback decomposition of $\partial B^{n}$. We define the boundary $\partial \mathbf{C}$ as a multiset (set with multiplicities) of oriented $(n-1)-$ cells

$$
\partial \mathbf{C}=\bigcup\left(\varphi\left(L_{i}\right),\left[\varphi \circ \varphi_{i}\right]\right)
$$

where the union is over all $(n-1)-$ cells $L_{i} \in L$, each taken with the natural orientation $\left[\varphi_{i}\right]$ defined by $(9-1)$.

It is easy to see, using Theorem 3.4, that this definition does not depend on the choice of characteristic map $\varphi$ in the equivalence class.

Note that by definition of a PLCW, for each $L_{i} \in L, \varphi\left(L_{i}\right)$ is an $(n-1)$-cell of $K$; however, the same $(n-1)-$ cell $D \in K$ can appear in $\partial C$ more than once, and possibly with different orientations. Note also that passing from the multisets to the abelian group generated by oriented cells, we get the usual definition of the boundary operator in the chain complex of a CW complex. However, for applications to topological field theory, the definition of the boundary as a multiset is much more useful.

Example 9.3 Let $C$ be the 2 -cell shown below. Then $\partial C=\{a, \bar{a}, b\}$, where $\bar{a}$ denotes $a$ with opposite orientation.

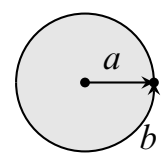

The proof of the following lemma is left to the reader as an exercise.

Lemma 9.4 Let $X$ be an oriented PL manifold with boundary and $K$ a PLCW decomposition of $X$. Then

$$
\bigcup_{\mathbf{C}} \partial \mathbf{C}=\left(\bigcup_{D} \mathbf{D}\right) \cup\left(\bigcup_{F} \mathbf{F} \cup \overline{\mathbf{F}}\right),
$$

where

- $C$ runs over all $n$-cells of $K$, each taken with orientation induced by orientation of $X$,

- $\quad D$ runs over all $(n-1)$ cells such that $D \subset \partial X$, each taken with orientation induced by orientation of $\partial X$,

- $F$ runs over all (unoriented) $(n-1)-$ cells such that $\stackrel{\circ}{F} \subset \operatorname{Int}(X) ; \mathbf{F}$ and $\overline{\mathbf{F}}$ are the two possible orientations of $F$. 


\section{References}

[1] B Balsam, J Kirillov, Alexander, Turaev-Viro invariants as an extended TQFT arXiv: 1004.1533

[2] R Oeckl, Renormalization of discrete models without background, Nuclear Phys. B 657 (2003) 107-138 MR1969693

[3] R Oeckl, Discrete gauge theory: From lattices to TQFT, Imperial College Press, London (2005) MR2174961

[4] C P Rourke, B J Sanderson, Introduction to piecewise-linear topology, Springer Study Edition, Springer, Berlin (1982) MR665919

Department of Mathematics, SUNY at Stony Brook

Stony Brook, NY 11794, USA

kirillov@math.sunysb.edu

http://www. math.sunysb.edu/ kirillov/

Received: 21 June 2011 